Supporting Information for

\title{
Resonance Raman Study of Shell Morphology in InP/ZnSe/ZnS Core/Shell/Shell Nanocrystals
}

Paul Cavanaugh, ${ }^{1}$ Ilan Jen-La Plante, ${ }^{2}$ Christian Ippen, ${ }^{2}$ Ruiqing Ma, ${ }^{2}$ David F. Kelley, ${ }^{1 *}$ and Anne Myers Kelley ${ }^{*}$

${ }^{1}$ Department of Chemistry and Biochemistry, University of California, Merced, 5200 North Lake Road, Merced, CA 95340

2 Nanosys Inc., 233 S. Hillview Dr, Milpitas, CA 95035

*David F. Kelley: e-mail dfkelley@ucmerced.edu, tel. (209) 228-4354. Anne Myers Kelley: e-mail amkelley@ucmerced.edu, tel. (209) 228-4345. 


\section{Wavefunction and charge distribution calculations for InP/ZnSe QDs}

The positive and negative charge distributions presented in Figure 2 were calculated from the effective mass approximation particle in a sphere model as described in refs. 1 and 2 using the following parameters: $\mathrm{InP}$ core radius $=1.5 \mathrm{~nm}$, ZnSe shell thickness $=2.5 \mathrm{~nm}$, electron effective mass $=0.14 \mathrm{~m}_{\mathrm{e}}$, hole effective mass $=0.60 \mathrm{~m}_{\mathrm{e}}$, core-shell band offsets $=0.4 \mathrm{eV}$ for conduction band and $1.0 \mathrm{eV}$ for valence band, bulk bandgap $=1.34 \mathrm{eV}$, and energy offsets of $3.0 \mathrm{eV}$ between the ligand/solvent environment and the conduction and valence bands of the shell. The effective masses were taken to be averages of the InP and ZnSe values because of the difficulties with nonHermitian Hamiltonians caused by assuming different masses, as discussed in ref. 2. The electron-hole interaction was then treated through a configuration interaction calculation using the two lowest-energy electron wavefunctions and the four lowest-energy hole wavefunctions (8 excitonic states). The electron and hole charge densities are plotted in Figure 2 as $\rho^{2}$ to account for the radial dependence of the volume element.

\section{Raman frequencies and intensities at $501.7 \mathrm{~nm}$}

Table S1. Peak fitting at $501.7 \mathrm{~nm}$ excitation. Estimated uncertainties are $\pm 1 \mathrm{~cm}^{-1}$ for the peak frequencies, \pm 1 for the low frequency to high frequency intensity ratio, and $\pm 20 \%$ differential Raman cross-section.

\begin{tabular}{|c|c|c|c|c|c|}
\hline sample & $\begin{array}{l}\text { ZnSe high } \\
\text { freq } / \mathrm{cm}^{-1}\end{array}$ & $\begin{array}{l}\text { InP high } \\
\text { freq } / \mathrm{cm}^{-1}\end{array}$ & $\begin{array}{l}\text { ZnSe low freq / high } \\
\text { freq int ratio }\end{array}$ & $\begin{array}{c}\mathrm{do} \text { ZnSe } / \mathrm{d} \Omega \\
/ 10^{-7} \AA^{2}\end{array}$ & $\begin{array}{l}{\left[\left(\mathrm{do}_{\mathrm{ZnSe}} / \mathrm{d} \Omega\right) / \mathrm{vol}\right.} \\
\mathrm{ZnSe}] \\
/ 10^{-10} \AA^{2} \mathrm{~nm}^{-3}\end{array}$ \\
\hline R0.99 & 244.4 & 353.1 & 3 & 0.58 & 2.2 \\
\hline R1.04 & 244.5 & 354.9 & 4 & 0.67 & 2.5 \\
\hline R1.09 & 246.1 & 354.1 & 4 & 0.26 & 1.8 \\
\hline R1.11 & 246.5 & 354.8 & 3 & 0.50 & 1.6 \\
\hline R1.48 & 244.3 & 352.8 & 6 & 0.35 & 2.6 \\
\hline R1.54 & 244.0 & 352.2 & 5 & 0.17 & 1.4 \\
\hline R1.58 & 247.3 & 354.0 & 3 & 0.41 & 2.4 \\
\hline R1.63 & 243.7 & 352.8 & 4 & 0.47 & 2.4 \\
\hline
\end{tabular}




\section{Resonance Raman depolarization ratios}

The resonance Raman depolarization ratio is given by ${ }^{3}$

$$
\rho=\frac{I_{\perp}}{I_{\|}}=\frac{5 \Sigma^{1}+3 \Sigma^{2}}{10 \Sigma^{0}+4 \Sigma^{2}}
$$

where the rotational invariants are given by

$$
\begin{aligned}
\Sigma^{0} & =\frac{1}{3}\left|\alpha_{x x}+\alpha_{y y}+\alpha_{z z}\right|^{2} \\
\Sigma^{1} & =\frac{1}{2}\left\{\left|\alpha_{x y}-\alpha_{y x}\right|^{2}+\left|\alpha_{x z}-\alpha_{z x}\right|^{2}+\left|\alpha_{y z}-\alpha_{z y}\right|^{2}\right\} \\
\Sigma^{2} & =\frac{1}{2}\left\{\left|\alpha_{x y}+\alpha_{y x}\right|^{2}+\left|\alpha_{x z}+\alpha_{z x}\right|^{2}+\left|\alpha_{y z}+\alpha_{z y}\right|^{2}\right\} \\
& +\frac{1}{3}\left\{\left|\alpha_{x x}-\alpha_{y y}\right|^{2}+\left|\alpha_{x x}-\alpha_{z z}\right|^{2}+\left|\alpha_{y y}-\alpha_{z z}\right|^{2}\right\}
\end{aligned}
$$

and the $\alpha_{i j}$ are the components of the Raman polarizability tensor in QD-fixed coordinates. Away from resonance, the polarizability tensor is symmetric $\left(\alpha_{i j}=\alpha_{j i}\right)$ so $\Sigma^{1}=0$. On resonance, $\Sigma^{1}$ is also usually zero although it can be nonzero in unusual cases involving degenerate electronic states.

Off resonance, the allowed components of the Raman polarizability tensor are determined by the symmetry properties of the QD and the phonon. For the Raman intensity to be nonzero, the product of the symmetry species of the phonon of interest and the pair of coordinates in the Raman tensor must contain the totally symmetric species. The products $x x, y y$, and zz always transform as the totally symmetric species, so $\Sigma^{0}$ must be zero for nontotally symmetric modes. Therefore only $\Sigma^{2}$ is nonzero and the depolarization ratio is $3 / 4$ ("depolarized" Raman transitions). For totally symmetric modes, both $\Sigma^{0}$ and $\Sigma^{2}$ may be nonzero and the depolarization ratio may range anywhere from 0 to $3 / 4$ ("polarized" transitions).

Here we further examine depolarization ratios in resonance Raman scattering. We assume that the Raman scattering is "A-term", that is the Condon approximation holds for the electronic 
transitions (coordinate dependence of the electronic transition dipole moment is neglected). This is usually a valid assumption when on resonance with strong electronic transitions. In this case, the elements of the polarizability tensor for fundamental scattering have the following forms: ${ }^{4}$

$$
\alpha_{j k}(\omega)=\sum_{e} \mu_{e g}^{j} \mu_{g e}^{k} \sum_{v} \frac{\langle 1 \mid v\rangle\langle v \mid 0\rangle}{\omega_{e g}+\omega_{v}-\omega-i \Gamma}
$$

where the sum is over electronic states (e) and phonon sublevels of those states (v), and $\mu_{e g}^{j}$ is the component of the $\mathrm{g} \rightarrow \mathrm{e}$ transition dipole moment in the QD-fixed $\mathrm{j}$ direction. Here we assume that a set of axes can be defined such that each excitonic transition is polarized along $\mathrm{x}, \mathrm{y}$, or $\mathrm{z}$, although this does not necessarily hold in systems of sufficiently low symmetry. In this case the polarizability tensor has only diagonal elements, $\alpha_{j j}$. In our previous work on CdSe we assumed $\alpha_{x x}=\alpha_{y y}$, i.e. one unique symmetry axis.1,5 Here we relax this assumption and allow all three directions to be nondegenerate.

In a system of perfectly spherical symmetry (an idealized QD), all three directions are equivalent and $\alpha_{x x}=\alpha_{y y}=\alpha_{z z}$ at all frequencies. Then, only $\Sigma^{0}$ is nonzero and the depolarization ratio is zero - that is, for any polarization direction of the applied radiation, the scattered light has the identical polarization. Once that spherical symmetry is broken, however, very different depolarization ratios may be observed. Consider the simplest case of an initially isotropic, single excitonic transition split into slightly nondegenerate $\mathrm{x}, \mathrm{y}$, and $\mathrm{z}$ components that each have the same transition dipole moment and exciton-phonon coupling strengths but slightly different energies. Eq. (S4) then becomes

$$
\alpha_{j j}(\omega)=\left|\mu_{e g}\right|^{2} \sum_{v}\langle 1 \mid v\rangle\langle v \mid 0\rangle \sum_{j} \frac{1}{\omega_{e g, j}+\omega_{v}-\omega-i \Gamma}
$$

where the sum is over $\mathrm{x}, \mathrm{y}$, and $\mathrm{z}$. The Raman depolarization ratios then depend entirely on the homogeneous linewidth $\Gamma$ and the energetic splittings among the $\mathrm{x}, \mathrm{y}$, and $\mathrm{z}$ components of the excitonic transition. 
Figure S1 shows calculated resonance Raman depolarization ratios as a function of the assumed splittings between the $\mathrm{x}, \mathrm{y}$, and $\mathrm{z}$ components, for a fixed value of the homogeneous linewidth $\Gamma=100 \mathrm{~cm}^{-1}$. The phonon frequency is set to $250 \mathrm{~cm}^{-1}$ and the exciton-phonon coupling is assumed very weak, such that only the $\mathrm{v}=0$ and $\mathrm{v}=1$ states must be considered in the sum in eq. (S4). The x-polarized transition was placed at $20000 \mathrm{~cm}^{-1}$ and the other two transitions were spaced to higher energies by the increments $\Delta$ given in the figure legend. When the splitting of the degeneracy is small compared to the linewidth (black curve), the depolarization ratios are close to the value of zero expected for an isotropic system. As the splitting increases the depolarization ratios increase, reaching a maximum value near 0.65 for a splitting of $200 \mathrm{~cm}^{-1}$ (red curve). As the splitting becomes even larger (blue curve), the depolarization ratios start to approach the value of $1 / 3$ for a single, nondegenerate electronic transition.

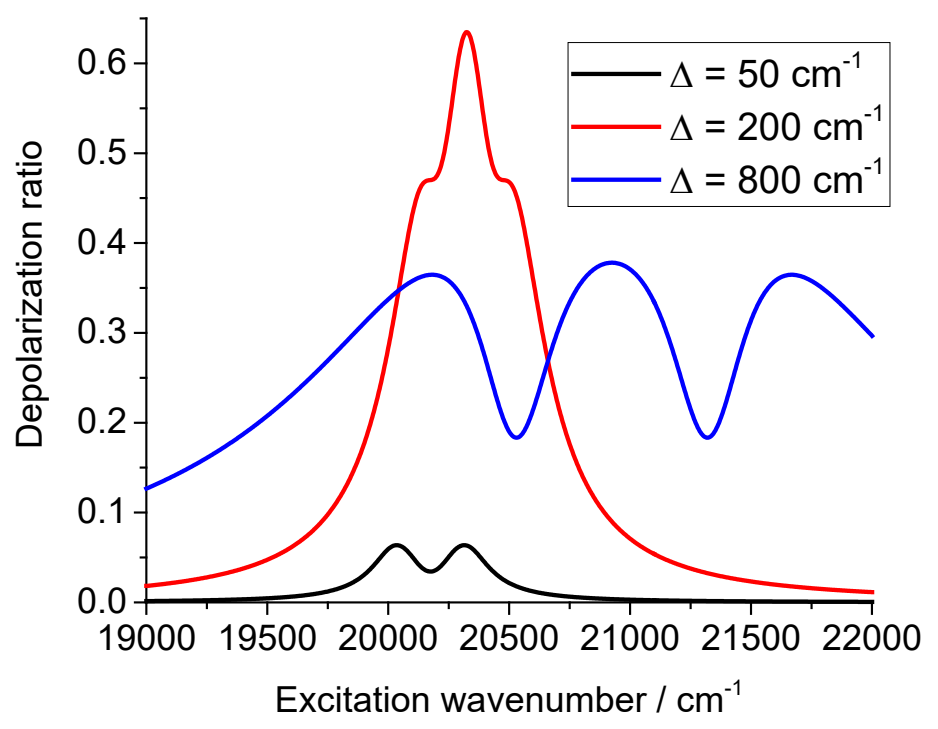

Figure S1. Calculated Raman depolarization ratios for a system with three otherwise identical excitonic transitions with mutually perpendicular polarizations and resonant energies split by the indicated amounts. The lowest-energy transition is at $20000 \mathrm{~cm}^{-1}$, the phonon frequency is $250 \mathrm{~cm}^{-1}$, and the excitonic homogeneous linewidth is $100 \mathrm{~cm}^{-1}$. 
These calculations are intended only to illustrate the wide range of depolarization ratios that may be expected even in a very simple model system. Our experimental structures have many different phonon modes, multiple overlapping excitonic transitions with different transition dipole moments and exciton-phonon coupling strengths, and significant inhomogeneous broadening, which will tend to smooth out the variations in depolarization ratio with excitation wavelength. Quantitative analysis of the depolarization ratios in such a complex system is beyond the scope of the current work.

\section{$\underline{\text { IV. Molar absorptivity determinations for InP/ZnSe/ZnS QDs }}$}

In order to determine the absolute Raman cross-section for the QDs, we need the QD concentration in the solution which we obtain from Beer's Law. The molar absorptivity of the QD is obtained following Method 2 in the SI of ref. 6. We assume that the optical constants of QDs are the same as those of the corresponding bulk materials at high photon energies, apart from local field factors. The bulk optical constants and the local field factors are used to calculate the theoretical absorption cross section at some energy sufficiently high that the effects of quantum confinement should not be important $-3.54 \mathrm{eV}(350 \mathrm{~nm})$ in this case. The experimental ratio of QD absorbances at high energy and at the lowest exciton is then used to determine the cross-section at the lowest exciton. For application to a core/shell system, we modified eq. (8) of $\mathrm{Kuno}^{7}$ to

$$
\sigma_{Q D}=\frac{4 \pi}{n_{m} \lambda}\left\{n_{c} k_{c} V_{c}+n_{s} k_{s} V_{s}\right\} \frac{9 n_{m}^{4}}{\left(n_{s}^{2}-k_{s}^{2}+2 n_{m}^{2}\right)^{2}+4\left(n_{s} k_{s}\right)^{2}}
$$

where " $c$ " is core, " $\mathrm{s}$ " is shell, and " $\mathrm{m}$ " is medium (the solvent cyclohexane), $n$ and $k$ are the real and imaginary parts of the complex refractive index, and $\mathrm{V}$ is the volume (of core or shell). The last term is the local field correction, for which we use the optical properties of the shell because 
most of the volume is in the shell. For cyclohexane near $350 \mathrm{~nm}, n_{m}$ is about $1.45 .{ }^{8}$ From Adachi, ${ }^{9}$ at $350 \mathrm{~nm}$ we have $n_{s}=2.85$ and $k_{s}=0.55$ for the ZnSe shell. From Aspnes and Studna, ${ }^{10}$ at $350 \mathrm{~nm}$ we have $n_{c}=3.17$ and $k_{c}=1.92$ for the InP core. The contribution from the very thin ZnS outer shell was neglected. Once we have used eq. (S5) to calculate the absorption cross-section at $350 \mathrm{~nm}$, the experimental ratio of QD absorbances at $350 \mathrm{~nm}$ and at the lowest exciton is used to determine the absorption cross-section, and thereby the molar absorptivity, at the lowest exciton. The results are given in the table below.

Table S2. Molar absorptivities for quantum dots.

\begin{tabular}{|c|c|c|c|c|c|}
\hline Sample & $\begin{array}{c}\text { InP volume / } \\
\mathrm{nm}^{3}\end{array}$ & $\begin{array}{c}\text { ZnSe volume } \\
/ \mathrm{nm}^{3}\end{array}$ & $\begin{array}{c}\sigma_{\mathrm{A}} \text { at 350 } \mathrm{nm} \\
/ \AA^{2}\end{array}$ & $\begin{array}{c}\sigma_{\mathrm{A}} \text { at peak of } \\
\text { lowest } \\
\text { exciton } / \AA^{2}\end{array}$ & $\begin{array}{c}\varepsilon \text { at peak of } \\
\text { lowest } \\
\text { exciton } \\
\mathrm{M}^{-1} \mathrm{~cm}^{-1}\end{array}$ \\
\hline R0.99 & 14.9 & 260 & 318 & 11.4 & $2.97 \times 10^{5}$ \\
\hline R1.04 & 15.9 & 265 & 327 & 10.3 & $2.68 \times 10^{5}$ \\
\hline R1.09 & 16.5 & 145 & 209 & 8.2 & $2.13 \times 10^{5}$ \\
\hline R1.11 & 15.9 & 319 & 381 & 9.3 & $2.42 \times 10^{5}$ \\
\hline R1.48 & 12.8 & 134 & 184 & 8.6 & $2.25 \times 10^{5}$ \\
\hline R1.54 & 12.8 & 122 & 172 & 9.4 & $2.44 \times 10^{5}$ \\
\hline R1.58 & 14.7 & 170 & 227 & 9.4 & $2.44 \times 10^{5}$ \\
\hline R1.63 & 12.8 & 199 & 249 & 9.6 & $2.51 \times 10^{5}$ \\
\hline
\end{tabular}

\section{Unsubtracted Raman spectra}

Figure S2 shows the resonance Raman spectra of sample R0.99 at the three excitation wavelengths prior to background subtraction. 

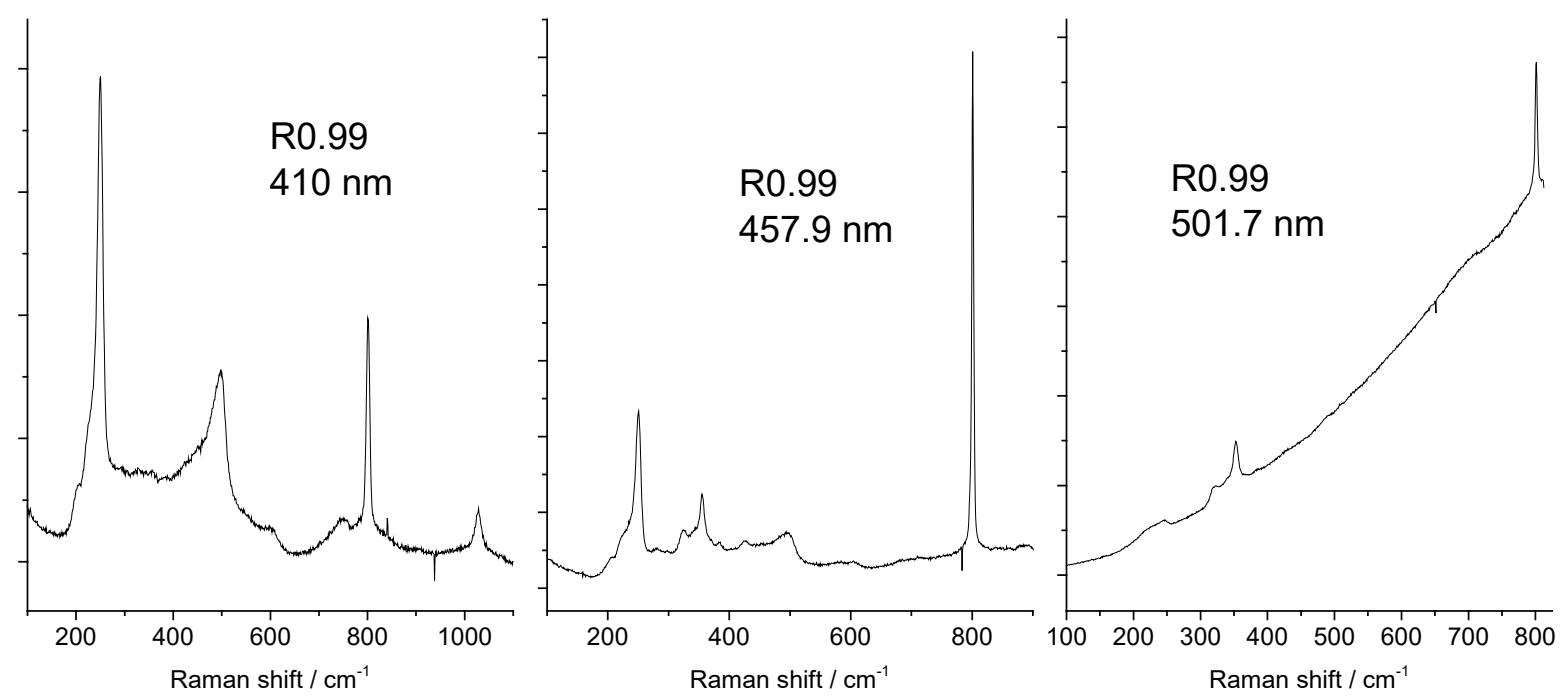

Figure S2. Unsubtracted resonance Raman spectra of R0.99 at the indicated excitation wavelengths.

1. Lin, C.; Gong, K.; Kelley, D. F.; Kelley, A. M., Size Dependent Exciton-Phonon Coupling in CdSe Nanocrystals Through Resonance Raman Excitation Profile Analysis. J. Phys. Chem. C 2015, 119, 7491-7498.

2. Lin, C.; Gong, K.; Kelley, D. F.; Kelley, A. M., Electron-Phonon Coupling in CdSe/CdS Core-Shell Quantum Dots. ACS Nano 2015, 9, 8131-8141.

3. Kelley, A. M.; Shoute, L. C. T.; Blanchard-Desce, M.; Bartholomew, G. P.; Bazan, G. C., Resonance Raman, Hyper-Raman, and Hyper-Rayleigh Depolarization Ratios and Symmetry Breaking in Solution. Mol. Phys. 2006, 104, 1239-1247.

4. Myers, A. B., Excited Electronic State Properties from Ground-State Resonance Raman Intensities. In Laser Techniques in Chemistry, Myers, A. B.; Rizzo, T. R., Eds. Wiley: New York, 1995; pp 325-384.

5. Baker, J. A.; Kelley, D. F.; Kelley, A. M., Resonance Raman and Photoluminescence Excitation Profiles and Excited-State Dynamics in CdSe Nanocrystals. J. Chem. Phys. 2013, 139, 024702. 
6. Gong, K.; Kelley, D. F.; Kelley, A. M., Resonance Raman Spectroscopy and ElectronPhonon Coupling in Zinc Selenide Quantum Dots. J. Phys. Chem. C 2016, 120, 29533-29539.

7. Giblin, J.; Kuno, M., Nanostructure Absorption: A Comparative Study of Nanowire and Colloidal Quantum Dot Absorption Cross Sections. J. Phys. Chem. Lett. 2010, 1, 3340-3348.

8. Kozma, I. Z.; Krok, P.; Riedle, E., Direct Measurement of the Group-Velocity Mismatch and Derivation of the Refractive-Index Dispersion for a Variety of Solvents in the Ultraviolet. J. Opt. Soc. Am. B 2005, 22, 1479-1485.

9. $\quad$ Adachi, S.; Taguchi, T., Optical Properties of ZnSe. Phys. Rev. B 1991, 43, 9569-9577.

10. Aspnes, D. E.; Studna, A. A., Dielectric Functions and Optical Parameters of Si, Ge, GaP, GaAs, GaSb, InP, InAs, and InSb from 1.5 to 6.0 eV. Phys. Rev. B 1983, 27, 985-1009. 